\title{
Return to contact sports after spinal surgery
}

\author{
Mark G. Burnett, M.D., AND Volker K. H. Sonntag, M.D. \\ Division of Neurological Surgery, Barrow Neurological Institute, St. Joseph's Hospital and Medical \\ Center, Phoenix, Arizona
}

\begin{abstract}
$\checkmark$ Decisions regarding the return of injured athletes to contact sports after spinal surgery can be complicated. The authors offer a brief overview of the return-to-play guidelines used successfully at their institution for the past two decades when caring for professional and amateur athletes after spinal surgery.
\end{abstract}

\author{
KEY WordS - cervical spine - thoracic spine - lumbar spine • sports medicine • \\ spinal surgery
}

$\mathrm{P}$ OSTOPERATIVE care of athletes after spinal surgery can be challenging. Athletes are often model patients, following doctors' orders faithfully and working hard in physical therapy. They are highly motivated to make a rapid recovery and to return to full participation in sports. Unfortunately, this desire to return to play can make a protracted rehabilitation period frustrating for both physician and patient. The decision to prohibit athletes from returning to play can have significant emotional and financial implications. Surgeons may feel pressure from patients (or their employers in the case of professional athletes) to shorten the customary postoperative period for rest or bracing. Surgeons also may be pressured to allow athletes to participate in sports after surgery when it is contraindicated. The current medicolegal climate further complicates decision making because fear of future litigation can prompt physicians to be overly conservative in their recommendations about returning to full competition.

Given the external pressures physicians must face regarding postoperative care and return-to-play restrictions, a discrete body of literature to aid decision making or at least a set of consensus guidelines developed by spinal surgeons to assist treating physicians would be beneficial. Unfortunately, there are no such guidelines, and most of the literature in this area has focused on return to play after spinal injury rather than on postoperative care issues. Consequently, there is little information to guide practitioners.

In this article we attempt to provide a framework to assist decision making with regard to return to play after spinal surgery. Because the literature is incomplete, we have integrated published research with the senior author's (V.K.H.S.) current practice paradigm for dealing with college and professional athletes. As a general matter of practice, athletes requiring a repeated operation or additional surgery in the same spinal region are absolutely prohibited from returning to contact sports. Therefore, the following discussion is limited to first-time surgery.

\section{Cervical Injuries}

Much has been written about sports injuries involving the cervical spine. Within this literature are recommenda- tions regarding the athlete's return to play after surgery, but the published data underlying these recommendations are scant. Furthermore, nontraumatic cervical disorders have received little attention. Therefore, physicians have had to extrapolate information from trauma cases and apply it to postoperative patients treated for nontraumatic conditions.

Torg and Ramsey-Emrhein ${ }^{4}$ have made perhaps the most significant contribution to the current treatment of cervical injuries in athletes. Their recommendations for returning to participation in contact sports are widely cited and are based on sound principles. These authors concluded that $\mathrm{C} 1-2$ (high cervical region) fusion is an absolute contraindication for returning to contact sports because the $\mathrm{C}-1$ arch is relatively fragile and stability depends on the status of the periodontoid ligaments. This recommendation is not based on specific statistical or outcome data. Nonetheless, the delicacy of the ligamentous and bone structures at $\mathrm{C} 1-2$ and the devastating consequences of a high cervical spinal cord injury make this conclusion logical, even when a fusion construct is deemed solid. The C1-2 motion segment is responsible for more than half of all cervical rotation. Therefore, athletes in whom this type of fusion was performed would be at a functional disadvantage that theoretically could increase their chances of subsequent injury on the field. Because this rotational immobility would result from any fusion involving the occipital region, we advocate that patients undergoing occipitocervical fusion or $\mathrm{C} 1-2$ fusion of any kind be restricted permanently from participation in contact sports.

Surgeries of the subaxial spine require individual consideration. Posterior cervical foraminotomies do not significantly weaken the spine, and a full return to contact sports is possible. We believe that patients who require more than a two-level laminectomy should be restricted from returning to full contact regardless of whether the laminectomy is augmented by lateral fusion or is freestanding. As more segments are included, multilevel laminectomy leads to increasing amounts of destabilization, due to loss of the dorsal tension band. Postlaminectomy kyphosis has been reported to be as high as $20 \%$ in pa- 
tients after laminectomy. ${ }^{2}$ Although lateral mass fusion increases the stiffness of the laminectomized region, junctional stresses above and below the rigid fusion are a concern. Laminoplasty has been suggested as a way to reduce the destabilizing effects of laminectomy without fusion. Like fusion, however, laminoplasty can lead to increased stiffness. ${ }^{3}$ In addition to these osseous and ligamentous biomechanical concerns, multilevel posterior cervical surgeries cause significant trauma to the paraspinal muscles. This trauma leads to denervation and atrophy that impair the spine's ability to support the extreme stresses sustained during contact sports.

The recommendations of Torg and Ramsey-Emrhein ${ }^{4}$ regarding return to contact sports after cervical injury and treatment with anterior surgery seem appropriate. Oneand two-level fusions are not absolute contraindications to participation in contact sports. Throughout his career, the senior author has used this guideline with success when dealing with professional football players. Anterior fusions of more than two levels would require permanent exclusion from contact sports due to concerns about junctional stresses around a lengthy construct and due to the restriction of movement that results from these surgeries. Single-level corpectomy will be considered to be equivalent to a two-level fusion for the purposes of this discussion. As with posterior foraminotomy, an anterior foraminotomy involves no fusion and is not destabilizing enough to contraindicate a full return to sports activities.

\section{Thoracic Injuries}

Little information is available about return to play after thoracic spinal surgery. Theoretically, the relative immobility of the region makes the stability of the thoracic spine significantly greater than that of the cervical spine after surgery. The thoracic spine gains significant stability from the rib cage, sternum, and broad paraspinal musculature. Biomechanical testing has shown that the osseous elements of the rib cage alone increase the stability of the thoracic spine by 20 to $40 \% .^{5}$ This added support is so great that some have even proposed that the ribs and sternum themselves should be included as a "fourth column" in the Denis three-column model of instability for thoracolumbar fractures. ${ }^{1}$ The additional support in this region is a key reason why our recommendations for returning to contact sports after thoracic surgery differ from those for the cervical region, despite the high ratio of spinal cord/ spinal canal diameter in the thoracic region.

The difficulty with broad consideration of the thoracic spine as a whole is that aside from its structural supportive role, it also serves as a transition segment from the cervical to the lumbar region. Therefore, many consider the thoracic spine as three separate but connected zones (midthoracic and two junctional zones). Moving caudally from the cervical region, the characteristics of the osseous structures shift progressively. The position of the facets begins to change from being coronally aligned in the high thoracic region to being sagittally aligned in the low thoracic region. Similarly, the size of the thoracic vertebral bodies increases as one moves caudally toward the lumbar region.

When the transition zones at the top and bottom of the thoracic spine are considered, it is clear that these regions are more vulnerable to injury than the midthoracic area. Consequently, the appropriate treatment for these areas is debatable. Particularly controversial is whether instrumentation needs to cross the transition zone completely or whether it is safe for a construct to end in the zone. Because the biomechanical profile changes markedly across these transition zones, we restrict athletes from returning to contact sports when they undergo an instrumented fusion that crosses the cervicothoracic or thoracolumbar junction. A natural corollary of this guideline is that patients with a fusion that terminates at a transition zone should also be prohibited from returning to contact play.

Patients who undergo thoracic decompression or fusion for the treatment of a disc herniation or trauma that does not involve the junctional segments are free to return to full participation in sports if they are pain free, are neurologically intact, and exhibit evidence of bone fusion on diagnostic imaging. This includes patients whose surgery spans the apex of the natural thoracic kyphosis, as long as sagittal and coronal alignment in this area is normal.

\section{Lumbar Injuries}

Perhaps because most lumbar spine conditions in athletes are muscular in origin and do not require surgical treatment, there has been little comprehensive research on return to play after lumbar surgery. Significant lumbar injuries sustained during competition are remarkably rare. When these injuries do occur, they are usually acute disc herniations. Initially, such injuries are treated conservatively. When required, discectomy procedures are well tolerated by athletes. ${ }^{6}$ If patients are pain free, there appears to be no contraindication to their returning to contact sports after discectomy. For athletes, as for other patients, a regimen of stretching and strengthening the abdominal and back muscles is a crucial aspect of the recovery process.

Athletes such as gymnasts and football linemen are prone to spondylolysis from repetitive axial loading of an extended lumbar spine. Treatment for this condition is usually conservative. However, pedicle screw fixation and fusion of the affected segment or direct repair by placing screws across the pars interarticularis may be needed when patients experience neurological deficits or intractable pain. When lumbar fusion surgery is performed, either with lateral fusion alone or with interbody fusion, we believe that there is no contraindication to patients returning to contact sports after complete recovery from surgery. Like patients with a thoracic injury, those with a lumbar injury should be pain free, have no disabling neurological deficit, and exhibit evidence of bone fusion on $\mathrm{x}-$ ray films before resuming play. A clear understanding between treating physicians and patients about the possibility and consequences of reinjury is crucial.

The rationale for allowing successfully treated athletes to return to contact sports after lumbar surgery is anatomically based. The spinal cord terminates at the top of the lumbar region, leaving only the spinal nerves to pass through the lumbar canal, which has a degree of tolerance to stenosis not present in the other areas of the spine. In the lumbar region, more anatomical narrowing is needed to produce neurological deficits compared with other regions. Furthermore, nerve root compression is much bet- 
M. G. Burnett and V. K. H. Sonntag

TABLE 1

Recommendations for return to contact sports after spinal surgery*

\begin{tabular}{|c|c|}
\hline Op Location/Procedure & Return to Contact Sports \\
\hline \multicolumn{2}{|l|}{ cervical } \\
\hline occiput-C2 region & no \\
\hline \multicolumn{2}{|l|}{$\begin{array}{l}\text { subaxial region } \\
\text { posterior }\end{array}$} \\
\hline posterior & \\
\hline \multicolumn{2}{|l|}{ foraminotomy } \\
\hline $\begin{array}{l}\text { smge-Iever } \\
\text { multilevel }\end{array}$ & yes \\
\hline & yes \\
\hline $\begin{array}{l}\text { fusion)/laminoplasty } \\
\text { single-level }\end{array}$ & \\
\hline 2-level & yes \\
\hline$>2$-level & no \\
\hline \multicolumn{2}{|l|}{ anterior } \\
\hline \multicolumn{2}{|l|}{ discectomy \& fusion/arthroplasty } \\
\hline single-level & yes \\
\hline 2-level & yes \\
\hline$>2$-level & no \\
\hline \multicolumn{2}{|l|}{ foraminotomy } \\
\hline single-level & yes \\
\hline multilevel & yes \\
\hline \multicolumn{2}{|l|}{ corpectomy } \\
\hline single-level & yes \\
\hline multilevel & no \\
\hline \multicolumn{2}{|l|}{ thoracic } \\
\hline \multicolumn{2}{|l|}{ midthoracic } \\
\hline $\mathrm{w} /$ deformity & yes \\
\hline w/o deformity & yes \\
\hline thoracolumbar junction zone & no \\
\hline \\
\hline \multicolumn{2}{|l|}{ discectomy/laminectomy/laminoplasty } \\
\hline single-level & yes \\
\hline multilevel & yes \\
\hline \multicolumn{2}{|l|}{ anterior or posterior fusion/arthroplasty } \\
\hline single-level & yes \\
\hline multilevel & yes \\
\hline
\end{tabular}

* To be considered eligible for a full return to activity, patients must be pain free, neurologically intact, and have completed an uneventful rehabilitative course.

ter tolerated than spinal cord compression. Therefore, we regard the lumbar spine as being similar to an extremity joint such as the knee, elbow, or ankle. These regions are all dynamic, highly stressed with activity, and surrounded by peripheral nerves. Spinal nerves do not have the anatomical freedom to escape compression in the same way that the ulnar nerve might when the elbow is fractured. However, the supportive muscular envelope gives the lumbar region a level of protection from injury far greater than that available to joints in the extremities.

\section{Conclusions}

Data on return to play for athletes after spinal surgery are incomplete. Therefore, surgeons must rely on sound judgment, using recommendations such as those provided in this article as a general guide. Advances in treatments such as minimally invasive surgical techniques and in other technologies may lessen the biomechanical aftereffects of spinal surgery and change some of the thinking about postoperative restrictions.

Concern about litigation may make extended restrictions or complete prohibition of return to play the path of least difficulty for surgeons. Nevertheless, physicians must not lose sight of the emotional and perhaps financial impact that an overly conservative medical decision may have on athletes. The role of the treating clinician is to recommend the best course for maintaining optimum health while avoiding an unnecessarily high risk of devastating injury. Therefore, physicians' recommendations regarding return to contact sports should be the same for both amateur and professional athletes. (See Table 1 for a summary of recommendations for return to contact sports after surgical procedures in various locations.) Because the livelihood of professional athletes depends on their ability to play, they may choose to disregard clinical advice and to accept a higher risk of injury than nonprofessional players. When athletes are allowed to return to sports, their progress should be tracked closely until they are fully reintegrated into their activities. Consistent counseling on the importance of the proper use and fitting of safety equipment cannot be overemphasized.

\section{References}

1. Berg EE: The sternal-rib complex. a possible fourth column in thoracic spine fractures. Spine 18:1916-1919, 1993

2. Kaptain GJ, Simmons NE, Replogle RE, Pobereskin L: Incidence and outcome of kyphotic deformity following laminectomy for cervical spondylotic myelopathy. J Neurosurg 93: 199-204, 2000

3. Ratliff JK, Cooper PR: Cervical laminoplasty: a critical review. J Neurosurg 98:230-238, 2003

4. Torg JS, Ramsey-Emrhein JA: Management guidelines for participation in collision activities with congenital, developmental, or postinjury lesions involving the cervical spine. Clin J Sport Med 7:273-291, 1997

5. Watkins R IV, Watkins R III, Williams L, Ahlbrand S, Garcia $\mathrm{R}$, Karamanian A, et al: Stability provided by the sternum and rib cage in the thoracic spine. Spine 30:1283-1286, 2005

6. Watkins RG IV, Williams LA, Watkins RG III: Microscopic lumbar discectomy results for 60 cases in professional and Olympic athletes. Spine J 3:100-105, 2003

Manuscript received July 18, 2006.

Accepted in final form September 7, 2006.

Address reprint requests to: Volker K. H. Sonntag, M.D., c/o Neuroscience Publications, Barrow Neurological Institute, 350 West Thomas Road, Phoenix, Arizona 85013. email: neuropub@ chw.edu. 